\title{
MOMENTS OF A MARKOV-MODULATED, IRREDUCIBLE NETWORK OF FLUID QUEUES
}

\author{
LANDY RABEHASAINA, ${ }^{*}$ ENSSAT
}

\begin{abstract}
We study a network of fluid queues in which exogenous arrivals are modulated by a continuous-time Markov chain. Service rates in each queue are proportional to the queue size, and the network is assumed to be irreducible. The queue levels satisfy a linear, vectorvalued differential equation. We obtain joint moments of the queue sizes recursively, and deduce the Laplace transform of the queue sizes in the stationary regime.
\end{abstract}

Keywords: Linear fluid network; Markov modulation

2000 Mathematics Subject Classification: Primary 60K25; 60K30

Secondary $60 \mathrm{~J} 25$

\section{Introduction and notation}

In this paper, we consider a network consisting of $N$ fluid queues of infinite capacity. The study of such networks is motivated by, among other things, high-speed telecommunications networks, where a possible application is a communications network in which large incoming messages are broken up into small packets (cells) and sent from origin to destination. For some literature about stochastic fluid networks, see [2, Chapter 7] for a thorough account, [4] and [7] for stability of such networks, and [6] and [8] for some calculations on the stationary regime of the network.

Let $Q^{1}(t), \ldots, Q^{N}(t)$ be the fluid content at time $t$. Fluid arrives in queue $j$ at a rate $\lambda^{j}(X(t))$, where $X(t)$ is a continuous-time Markov chain, and is processed at a rate, $\mu^{j}\left(Q^{j}(t)\right)$, depending on the state. A reasonable assumption would be that $\mu^{j}(\cdot)$ is a nondecreasing function, i.e. the bigger the size of the queue, the bigger the service rate. In fact, we suppose in this paper that $\mu^{j}\left(Q^{j}(t)\right)$ is of the form $\mu^{j} Q^{j}(t)$ for $\mu^{j}>0$; the service rate is then linear with respect to the queue size. After being processed, a fraction $p_{j i}$ of fluid goes from queue $j$ to queue $i(i \in\{1, \ldots, N\})$. Thus, $1-\sum_{i \neq j} p_{j i}$ is, in particular, less than 1 and greater than 0 , and represents the fraction of fluid leaving the network from queue $j$.

Stochastic properties of a network of fluid queues where service or arrival rate depend on the fluid level and the state of an underlying Markov process have already been studied (see, e.g. [5]). However, our fluid network model is specifically the one studied in [8] and [6], with some minor differences. More precisely, in [8] the input rate is given by a general $N$-dimensional, nondecreasing process $A(t)=\left(A^{1}(t), \ldots, A^{N}(t)\right.$ ) (of which increments in our situation would thus be given by $\left.\mathrm{d} A^{j}(t)=\lambda^{j}(X(t)) \mathrm{d} t\right)$. In [6] an even more general model, featuring Lévy inputs modulated by a Markov chain, was studied.

The model here is also different in that the fractions of fluid $p_{i j}, i, j \in\{1, \ldots, N\}^{2}$, and the $\mu^{j}, j \in\{1, \ldots, N\}$, do not, unlike in [6], depend on $X(t)$. Processing and routeing within the network is thus deterministic, the only random part being the exogenous arrivals. This could

Received 10 August 2004; revision received 5 January 2006.

* Postal address: ENSSAT, 6 rue de Kerampont, BP 805, 22305 Lannion, France.

Email address: landy.rabehasaina@enssat.fr 
be illustrated in applications by a network within which random fluctuations occur on a shorter time scale than do the random fluctuation in the external inputs. In fact, introducing randomness in the $p_{i j}$ and the $\mu^{j}$ would make the analysis and formulae even more complicated.

However, in this paper we give an explicit recursion of all joint moments of the queue sizes in the stationary regime under an irreducibility assumption on the network, unlike in [8] and [6], where at best the first and second moments were given.

The model described here can be seen as the generalization of the single-fluid queue with linear service rate described in [1], [11], and [10], although [1] deals with a Lévy arrival process and the model in [10] features a white noise factor. More precisely, the vector of queue levels, $Q(t)=\left(Q^{1}(t), \ldots, Q^{N}(t)\right)^{\top}$ (the superscript 'T' denoting transposition), satisfies the differential equation

$$
\mathrm{d} Q(t)=\left(\lambda(X(t))-\left(I-P^{\top}\right) D_{\mu} Q(t)\right) \mathrm{d} t
$$

(see [8] and [6]), where $\lambda(X(t))=\left(\lambda^{1}(X(t)), \ldots, \lambda^{N}(X(t))\right)^{\top}$ is the vector of arrival rates, $D_{\mu}=\operatorname{diag}\left(\mu^{1}, \ldots, \mu^{N}\right), P=\left(p_{i j}\right)_{(i, j) \in\{1, \ldots, N\}^{2}}$ is commonly called the routeing matrix, and $I$ is the identity matrix. We let

$$
A:=\left(I-P^{\top}\right) D_{\mu}=\left(a_{i j}\right)_{(i, j) \in\{1, \ldots, N\}^{2}},
$$

implying that $Q(t)$ satisfies

$$
\mathrm{d} Q(t)=(\lambda(X(t))-A Q(t)) \mathrm{d} t .
$$

Now we give some assumptions and notation. We suppose the following to hold.

- The routeing matrix, $P$, is substochastic, i.e. $P^{n} \rightarrow 0$ as $n \rightarrow \infty$, or, equivalently, $I-P$ is an $M$-matrix, i.e. is invertible with nonnegative inverse (see [2, p. 164]). This can be expressed by the sum of the coefficients in each row of $P$ being less than or equal to 1 , and the sum of the coefficients in at least one row of $P$ being less than 1 .

- $\mu^{j}>0$ for all $j=1, \ldots, N$.

- $\{X(t), t \in \mathbb{R}\}$ is a stationary ergodic Markov chain on a finite state space $S=\{1, \ldots, K\}$ and has generator matrix $Q=\left(q_{i j}\right)_{i, j \in S \times S}$ and distribution $\pi=\left(\pi_{1}, \ldots, \pi_{K}\right)$.

We also introduce the reversed Markov chain $X^{*}(t)=X\left((-t)^{-}\right)$, whose infinitesimal generator, $Q^{*}=\left(q_{i j}^{*}\right)_{i j \in S \times S}$, satisfies $q_{i j}^{*}=q_{j i} \pi_{j} / \pi_{i}$.

One of the advantages of our model is that it yields explicit expressions for the queue content as well as for the random variable towards which it converges in distribution. More precisely, we have the following proposition.

Proposition 1.1. The solution, $(Q(t))_{t \geq 0}$, of $(1.1)$ satisfies

$$
Q(t)=\exp (-A t) Q(0)+\int_{0}^{t} \exp (-A(t-s)) \lambda(X(s)) \mathrm{d} s .
$$

Furthermore, $Q(t)$ converges in distribution, independently of the initial conditions, to

$$
W=\left(W_{1}, \ldots, W_{N}\right)^{\top}:=\int_{-\infty}^{0} \exp (A s) \lambda(X(s)) \mathrm{d} s,
$$


which, by making the change of variable $s \rightarrow-s$, equals

$$
W=\int_{0}^{\infty} \exp (-A s) \lambda\left(X^{*}(s)\right) \mathrm{d} s
$$

Proof. The expressions for $Q(t)$ and $W$ are standard and come, for example, from Theorem 4.3 of [8]. The fact that the convergence in distribution holds independently of the value of $Q(0)$ comes from Theorem 4.2 of [8].

We define the Laplace transform of $W$ given $X^{*}(0)$ as follows: for all $i \in S$ and $u=$ $\left(u_{1}, \ldots, u_{N}\right)^{\top} \in(-\infty, 0]^{N}$,

$$
\phi_{i}(u):=\mathrm{E}\left(\exp \left(u^{\top} W\right) \mid X^{*}(0)=i\right)=\mathrm{E}\left(\exp \left(\sum_{k=1}^{N} u_{k} W_{k}\right) \mid X^{*}(0)=i\right) .
$$

Let $\phi(u):=\left(\phi_{1}(u), \ldots, \phi_{K}(u)\right)^{\top}$. We also let

$$
\Lambda(l):=\operatorname{diag}\left(\lambda^{1}(l), \ldots, \lambda^{N}(l)\right) \text { for all } l \in S .
$$

Furthermore, by $P^{*}$ we denote the transition probability matrix of the uniformized discrete-time Markov chain associated with $X^{*}$. We then have $P^{*}=I+Q^{*} / v$, where $v$ is the uniformization rate, satisfying $v \geq \max \left\{-q_{i, i}^{*}, i \in S\right\}$. Finally, we let $\mathcal{N}:=\{1, \ldots, N\}$. Recall that $N$ is the number of queues in the network and is fixed throughout the paper.

The paper is organized in the following way. In Section 2 we give an integral and a differential equation satisfied by the Laplace transform of $W$, using the Markov nature of $X(t)$. In Section 3 we more specifically suppose that $P$ is irreducible, i.e. that a part of the fluid leaving from one queue travels through the network to reach any other queue. We then obtain the joint moments of the queue sizes, $W_{1}, \ldots, W_{N}$, in the stationary regime; the Laplace transform is then easily deduced.

\section{Equations satisfied by the Laplace transform}

Similarly to the method used in [1] and [6], we give an integral equation satisfied by the Laplace transform of $W$. This is done using a uniformization technique for the Markov chain $X(t)$. Let us recall that $v$ is greater than $\max \left\{-q_{i, i}^{*}, i \in S\right\}$ and can be taken to be as large as we want.

Proposition 2.1. For $u \in(-\infty, 0]^{N}, \phi$ satisfies

$$
\phi(u)=\int_{0}^{\infty} \Delta(u, x) P^{*} \phi\left(\exp \left(-A^{\top} x\right) u\right) v \exp (-v x) \mathrm{d} x,
$$

where $\Delta(u, x)$ is the diagonal $K \times K$ matrix defined by

$$
\Delta(u, x)=\operatorname{diag}\left(\exp \left(u^{\top}(I-\exp (-A x)) A^{-1} \lambda(1)\right), \ldots, \exp \left(u^{\top}(I-\exp (-A x)) A^{-1} \lambda(K)\right)\right) .
$$

The integral equation satisfied by $\phi$ can be compared with Equation (5) of [6].

Proof of Proposition 2.1. Let $T_{1}$ be the first instant of jump of the uniformized Markov chain. $T_{1}$ is exponentially distributed with rate $v$, and we have

$$
\mathrm{d} P\left(X^{*}\left(T_{1}\right)=j, T_{1}=x \mid X^{*}(0)=i\right)=p_{i j}^{*} v \mathrm{e}^{-v x} \mathrm{~d} x .
$$


Given $T_{1}$ and $X^{*}\left(T_{1}\right)$ (the value of the Markov chain after it has changed state for the first time), we write

$$
\begin{aligned}
\phi_{i}(u)= & \sum_{j \in S} \int_{0}^{\infty} \mathrm{E}\left(\exp \left(u^{\top} W\right) \mid T_{1}=x, X^{*}\left(T_{1}\right)=j, X^{*}(0)=i\right) \\
& \times \mathrm{d} P\left(X^{*}\left(T_{1}\right)=j, T_{1}=x \mid X^{*}(0)=i\right) \\
= & \sum_{j \in S} \int_{0}^{\infty} \mathrm{E}\left(\exp \left(u^{\top} W\right) \mid T_{1}=x, X^{*}\left(T_{1}\right)=j, X^{*}(0)=i\right) v \exp (-v x) p_{i j}^{*} \mathrm{~d} x .
\end{aligned}
$$

Let $\Psi(x, j, i)=\mathrm{E}\left(\exp \left(u^{\top} W\right) \mid T_{1}=x, X^{*}\left(T_{1}\right)=j, X^{*}(0)=i\right)$. Using the expression of $W$, from the Markov property of $X^{*}$ we have

$$
\begin{aligned}
\Psi(x, j, i)= & \mathrm{E}\left(\exp \left(u^{\top} \int_{0}^{x} \exp (-A s) \lambda\left(X^{*}(s)\right) \mathrm{d} s+u^{\top} \int_{x}^{\infty} \exp (-A s) \lambda\left(X^{*}(s)\right) \mathrm{d} s\right)\right. \\
= & \exp \left(u^{\top}(I-\exp (-A x)) A^{-1} \lambda(i)\right) \\
& \times \mathrm{E}\left(\exp \left(u^{\top} \int_{x}^{\infty} \exp (-A s) \lambda\left(X^{*}(s)\right) \mathrm{d} s\right) \mid X^{*}\left(T_{1}\right)=j, X^{*}(0)=i\right)
\end{aligned}
$$

Now, by the homogeneity of $X^{*}$,

$$
\begin{aligned}
\Psi(x, j, i)= & \exp \left(u^{\top}(I-\exp (-A x)) A^{-1} \lambda(i)\right) \\
& \times \mathrm{E}\left(\exp \left(u^{\top} \exp (-A x) \int_{0}^{\infty} \exp (-A s) \lambda\left(X^{*}(s+x)\right) \mathrm{d} s\right) \mid X^{*}(x)=j\right) \\
= & \exp \left(u^{\top}(I-\exp (-A x)) A^{-1} \lambda(i)\right) \\
& \times \mathrm{E}\left(\exp \left(\left[\exp \left(-A^{\top} x\right) u\right]^{\top} \int_{0}^{\infty} \exp (-A s) \lambda\left(X^{*}(s)\right) \mathrm{d} s\right) \mid X^{*}(0)=j\right) \\
= & \exp \left(u^{\top}(I-\exp (-A x)) A^{-1} \lambda(i)\right) \phi_{j}\left(\exp \left(-A^{\top} x\right) u\right) .
\end{aligned}
$$

Combining this equality with (2.2) yields (2.1).

Proposition 2.1 yields the differential equation satisfied by $\phi$, as follows.

Proposition 2.2. The Laplace transform $\phi$ satisfies the following differential equation for $u \in$ $(-\infty, 0]^{N}$ :

$$
\nabla \phi(u) A^{\top} u=\left(F(u)+Q^{*}\right) \phi(u),
$$

where $F(u):=\operatorname{diag}\left(u^{\top} \lambda(1), \ldots, u^{\top} \lambda(K)\right)$ and $\nabla \phi(u)$ is the gradient of $\phi$, i.e. the $K \times N$ matrix whose $(i, j)$ th element, $i \in S, j \in \mathcal{N}$, is $\partial_{j} \phi_{i}(u)$.

Proof. Since $P^{*}=I+Q^{*} / \nu,(2.1)$ can be written as

$$
\begin{array}{r}
v\left(\phi(u)-\int_{0}^{\infty} \Delta(u, x) \phi\left(\exp \left(-A^{\top} x\right) u\right) v \exp (-v x) \mathrm{d} x\right) \\
=\int_{0}^{\infty} \Delta(u, x) Q^{*} \phi\left(\exp \left(-A^{\top} x\right) u\right) v \exp (-v x) \mathrm{d} x
\end{array}
$$


which, by changing variable according to $x \rightarrow x / v$, reads

$$
\begin{array}{r}
v\left(\phi(u)-\int_{0}^{\infty} \Delta\left(u, \frac{x}{v}\right) \phi\left(\exp \left(-A^{\top} \frac{x}{v}\right) u\right) \exp (-x) \mathrm{d} x\right) \\
=\int_{0}^{\infty} \Delta\left(u, \frac{x}{v}\right) Q^{*} \phi\left(\exp \left(-A^{\top} \frac{x}{v}\right) u\right) \exp (-x) \mathrm{d} x
\end{array}
$$

However, (2.4) reads

$$
\begin{aligned}
& v\left(\phi(u)-\int_{0}^{\infty} \phi\left(\exp \left(-A^{\top} \frac{x}{v}\right) u\right) \exp (-x) \mathrm{d} x\right) \\
& -v \int_{0}^{\infty}\left(\Delta\left(u, \frac{x}{v}\right)-I\right) \phi\left(\exp \left(-A^{\top} \frac{x}{v}\right) u\right) \exp (-x) \mathrm{d} x \\
& \quad=\int_{0}^{\infty} \Delta\left(u, \frac{x}{v}\right) Q^{*} \phi\left(\exp \left(-A^{\top} \frac{x}{v}\right) u\right) \exp (-x) \mathrm{d} x
\end{aligned}
$$

Letting $v \rightarrow \infty$ in (2.5), from standard convergence results we obtain

$$
\int_{0}^{\infty} \nabla \phi(u) A^{\top} u x \exp (-x) \mathrm{d} x-\int_{0}^{\infty} \partial_{2} \Delta(u, 0) x \phi(u) \exp (-x) \mathrm{d} x=Q^{*} \phi(u),
$$

where $\partial_{2} \Delta(u, 0)$ denotes the derivative of $\Delta$ with respect to the second variable, evaluated at $(u, 0)$. Since

$$
\partial_{2} \Delta(u, 0)=\operatorname{diag}\left(u^{\top} \lambda(1), \ldots, u^{\top} \lambda(K)\right)=F(u) \quad \text { and } \quad \int_{0}^{\infty} x \exp (-x) \mathrm{d} x=1,
$$

(2.6) yields (2.3).

\section{Distribution of the queue levels in the stationary regime}

Neither (2.1) nor (2.3) has a unique solution in general, at least not without imposing further conditions on $\phi$. However, we can obtain the moments of $W$ from (2.3).

We suppose in this section that the routeing matrix of the network $P$ is irreducible. In other words, it is possible for some fluid leaving any queue $i$ to reach any other queue $j$. The reason for this assumption will become clear in the computation of the moments of $W$.

Before studying the limiting distribution, we need some more notation. First, for all $n \in \mathbb{N}$, $\left(l_{1}, \ldots, l_{n}\right) \in\{1, \ldots, N\}^{n}=\mathcal{N}^{n}$, and $i \in S$, we let

$$
m_{i}^{n}\left(l_{1}, \ldots, l_{n}\right):=\mathrm{E}\left(W_{l_{1}} \cdots W_{l_{n}} \mid X^{*}(0)=i\right) .
$$

We also let

$$
m^{n}\left(l_{1}, \ldots, l_{n}\right):=\left(m_{1}^{n}\left(l_{1}, \ldots, l_{n}\right), \ldots, m_{K}^{n}\left(l_{1}, \ldots, l_{n}\right)\right)^{\top}
$$

and

$$
m^{n}:=\left\{m^{n}\left(l_{1}, \ldots, l_{n}\right),\left(l_{1}, \ldots, l_{n}\right) \in \mathcal{N}^{n}\right\} .
$$

The latter is a family of column vectors indexed in $\{1, \ldots, N\}^{n}$, and represents the joint moments of $W$ of order $n$. For instance, $m^{n}(j, \ldots, j)$ is the $n$th moment of queue $j$ :

$$
m^{n}(j, \ldots, j)=\left(\mathrm{E}\left(W_{j}^{n} \mid X^{*}(0)=1\right), \ldots, \mathrm{E}\left(W_{j}^{n} \mid X^{*}(0)=K\right)\right) .
$$


The definition of $m^{n}$ is not valid for $n=0$; however, in what follows $m^{0}$ will be identified with the column $K$-vector $(1, \ldots, 1)^{\top}$ (corresponding to the moment of $W$ given $X^{*}(0)=i$, $i=1, \ldots, K$, of order 0$)$.

Furthermore,

$$
A^{(n)}=\left(a_{\left(l_{1}, \ldots, l_{n}\right),\left(k_{1}, \ldots, k_{n}\right)}^{(n)}\right)_{\left(\left(l_{1}, \ldots, l_{n}\right),\left(k_{1}, \ldots, k_{n}\right)\right) \in \mathcal{N}^{n} \times \mathcal{N}^{n}}
$$

is the $N^{n} \times N^{n}$ matrix defined by

$$
a_{\left(l_{1}, \ldots, l_{n}\right),\left(k_{1}, \ldots, k_{n}\right)}^{(n)}= \begin{cases}\sum_{i=1}^{n} a_{l_{i} l_{i}} & \text { if }\left(l_{1}, \ldots, l_{n}\right)=\left(k_{1}, \ldots, k_{n}\right), \\ a_{l_{i} k_{i}} & \text { if } l_{j}=k_{j}, j \neq i, \text { and } l_{i} \neq k_{i}, \\ 0 & \text { otherwise },\end{cases}
$$

and for all $n \in \mathbb{N}$ we define the $\left(N^{n} \times K\right) \times\left(N^{n-1} \times K\right)$ block matrix

$$
\Lambda^{(n)}=\left(b_{\left(l_{1}, \ldots, l_{n}\right),\left(k_{1}, \ldots, k_{n-1}\right)}^{(n)}\right)_{\left(\left(l_{1}, \ldots, l_{n}\right),\left(k_{1}, \ldots, k_{n-1}\right)\right) \in \mathcal{N}^{n} \times \mathcal{N}^{n-1}},
$$

where each $b_{\left(l_{1}, \ldots, l_{n}\right),\left(k_{1}, \ldots, k_{n-1}\right)}^{(n)}$ is a $K \times K$ matrix defined by

$$
b_{\left(l_{1}, \ldots, l_{n}\right),\left(k_{1}, \ldots, k_{n-1}\right)}^{(n)}:= \begin{cases}\operatorname{diag}\left(\lambda^{k_{i}}(1), \ldots, \lambda^{k_{i}}(K)\right) & \text { if } l_{p}=k_{p} \text { for } p=1, \ldots, i-1 \\ & \text { and } l_{p+1}=k_{p} \text { for } p=i, \ldots, n-1 \\ 0 & \text { otherwise. }\end{cases}
$$

Finally, by $I_{S}$ and $I_{\mathcal{N}^{n}}$ we respectively denote the $K \times K$ and $N^{n} \times N^{n}$ identity matrices.

Remark 3.1. The matrix $A^{(n)}$ has the property that on one of its rows, row $\left(l_{1}, \ldots, l_{n}\right)$ say, the only entries which can be different from 0 are those corresponding to columns $\left(k_{1}, \ldots, k_{n}\right)$ equal (in index) to $\left(l_{1}, \ldots, l_{n}\right)$ and to columns $\left(k_{1}, \ldots, k_{n}\right)$ where one (and only one) of the $k_{i}$ differs from the corresponding $l_{i}$.

Let us also remember that if $M=\left(m_{i j}\right)$ is a $d \times d$ matrix and $N$ is a $p \times p$ matrix, their Kronecker product, $M \otimes N$, is the $(d \times p) \times(d \times p)$ matrix

$$
M \otimes N=\left(\begin{array}{ccc}
m_{11} N & \cdots & m_{1 d} N \\
\vdots & \ddots & \vdots \\
m_{d 1} N & \cdots & m_{d d} N
\end{array}\right)
$$

(see, e.g. [3, Chapter 3])

In the following we say that a matrix $R$ satisfies condition (C1) if

- $R=D_{R}-C_{R}$, where $D_{R}$ is a diagonal matrix with positive diagonal elements and $C_{R}$ is a matrix with $0 \mathrm{~s}$ on its diagonal and nonnegative off-diagonal elements; and

- the sum of the elements in each row of $R$ is nonnegative and the sum of the elements in at least one row is positive. 
Thus, if $R$ satisfies condition (C1), it can be interpreted as the infinitesimal generator matrix of an irreducible, continuous-time Markov chain with an absorbing state. We will say that $R$ satisfies condition $(\mathrm{C} 2)$ if

- $R$ satisfies (C1), and

- $C_{R}$ is irreducible.

In what follows, for any matrix, $R$, with the decomposition $R=D_{R}-C_{R}$, we will sometimes say (in an abuse of terminology) that $R$ is irreducible, instead of saying that $C_{R}$ is irreducible, as no confusion is possible.

Let us recall the graphical interpretation of the irreducibility of a matrix. The matrix $C_{R}$ is irreducible if its corresponding graph $g$ is connex. Let us recall that if $C_{R}=\left(c_{i j}\right)$ is a nonnegative $d \times d$ matrix, we define its corresponding graph $g$ on $\{1, \ldots, d\}$ by joining two points, $i$ and $j, i \neq j$, in $\{1, \ldots, d\}$, if and only if $c_{i j}>0$. Thus, if $C_{R}$ is irreducible, there exists between any $i$ and $j$ in $\{1, \ldots, d\}$ a path,

$$
i=p_{0} \rightarrow p_{1} \rightarrow \cdots \rightarrow p_{l-1} \rightarrow p_{l}=j,
$$

of length $l$, where $p_{1}, \ldots, p_{l-1}$ are in $\{1, \ldots, d\}$ and satisfy

$$
p_{k} \neq p_{k+1}, \quad c_{p_{k}, p_{k+1}}>0 .
$$

A property of irreducible matrices is that a matrix $C_{R}$ is irreducible if and only if its transpose is irreducible.

We can now present the following lemma, which is proved in, e.g. Lemma 2.2.1 of [9].

Lemma 3.1. Let $R$ be a matrix satisfying condition (C2). Then $R$ is invertible.

We will give the different moments of $W$ in two cases. First, we will consider the particular case in which $A$ satisfies condition (C1). Note that since $P$ is a routeing matrix in this paper, only $A^{\top}=D_{\mu}(I-P)$ satisfies condition (C1), not $A$ (unless $P$ is symmetric). Moreover, let us remark that since $P$ is irreducible from now on, if $A$ satisfies condition (C1) then it also satisfies condition (C2). However, it is the assumption on the sum of the rows of $A$ that is important, which is why we will talk about condition $(\mathrm{C} 1)$ rather than about condition $(\mathrm{C} 2)$ in this context. The moments of $W$ will be given in the general case in Section 3.2, in which results from Section 3.1 will be extensively used.

\subsection{A satisfies condition (C1)}

Lemma 3.2. Let us suppose that A satisfies condition (C1). Then $A^{(n)}$ satisfies condition $(C 2)$. In particular, $A^{(n)}$ is invertible, by Lemma 3.1.

Proof. Let us first consider the matrix $A$, and remember that $I-P$ is an $M$-matrix. It is easy to check that $A=\left(I-P^{\top}\right) D_{\mu}$ has the decomposition $A=D_{A}-C_{A}$ of condition $(\mathrm{C} 1)$, with $D_{A}=D_{\mu}$ and $C_{A}=P^{\top} D_{\mu}$. The matrix $C_{A}$ is irreducible because $P$ is irreducible. Now, since $A$ satisfies condition (C1), the sum of the elements in each row of $A$ is nonnegative and the sum of the elements in at least one row is positive. Denoting by $i_{0}$ this particular row, we have

$$
\begin{aligned}
\sum_{k \in \mathcal{N}} a_{i k} & \geq 0, \quad i \neq i_{0}, \\
\sum_{k \in \mathcal{N}} a_{i_{0}} & >0 .
\end{aligned}
$$


Let us now consider $A^{(n)}$. From (3.1), it is easy to check that $A^{(n)}$ is a matrix with positive diagonal elements and nonpositive off-diagonal elements (remember that in (3.1), $a_{l_{i} l_{i}}>0$ and $a_{l_{i} k_{i}} \leq 0$ if $l_{i} \neq k_{i}$ ). We then have the decomposition $A^{(n)}=D_{A^{(n)}}-C_{A^{(n)}}$ of condition (C1). From (3.1) we see that if $\left(l_{1}, \ldots, l_{n}\right)$ and $\left(k_{1}, \ldots, k_{n}\right)$ are in $\mathcal{N}^{n}$, then

$$
a_{\left(l_{1}, \ldots, l_{n}\right),\left(k_{1}, \ldots, k_{n}\right)}^{(n)}=0
$$

if $\left(k_{1}, \ldots, k_{n}\right)$ is not equal to $\left(l_{1}, \ldots, l_{n}\right)$ or differs in more than one component. Thus, by summing the elements of row $\left(l_{1}, \ldots, l_{n}\right)$ of $A^{(n)}$, from (3.1) we obtain

$$
\begin{aligned}
\sum_{\left(k_{1}, \ldots, k_{n}\right) \in \mathcal{N}^{n}} a_{\left(l_{1}, \ldots, l_{n}\right),\left(k_{1}, \ldots, k_{n}\right)}^{(n)} & =\sum_{i=1}^{n} a_{l_{i} l_{i}}+\sum_{i=1}^{n} \sum_{k_{i} \neq l_{i}} a_{l_{i} k_{i}} \\
& =\sum_{i=1}^{n}\left(a_{l_{i} l_{i}}+\sum_{k_{i} \neq l_{i}} a_{l_{i} k_{i}}\right) \\
& =\sum_{i=1}^{n}\left(\sum_{k_{i} \in \mathcal{N}} a_{l_{i} k_{i}}\right) .
\end{aligned}
$$

Now, from (3.2) we have $\sum_{k_{i} \in \mathcal{N}} a_{l_{i} k_{i}} \geq 0$ for all $l_{i}$. Thus,

$$
\sum_{\left(k_{1}, \ldots, k_{n}\right) \in \mathcal{N}^{n}} a_{\left(l_{1}, \ldots, l_{n}\right),\left(k_{1}, \ldots, k_{n}\right)}^{(n)} \geq 0 .
$$

Moreover, from (3.2) and (3.3) we see that

$$
\sum_{\left(k_{1}, \ldots, k_{n}\right) \in \mathcal{N}^{n}} a_{\left(l_{1}, \ldots, l_{n}\right),\left(k_{1}, \ldots, k_{n}\right)}^{(n)}>0
$$

whenever one of the $l_{j}$ is equal to $i_{0}$. It follows that $A^{(n)}$ satisfies condition (C1).

To prove that $A^{(n)}$ satisfies condition (C2), it remains to prove that $C_{A^{(n)}}$ is irreducible. This amounts to showing that the graph on $\mathcal{N}^{n}$ associated to $C_{A^{(n)}}, g^{A^{(n)}}$, is connex. We know that $C_{A}$ is irreducible, i.e. that the graph, $g$, on $\mathcal{N}$ associated to $C_{A}$ is connex. Hence, from any point $k \in \mathcal{N}$ there is a path

$$
k=p_{0} \rightarrow p_{1} \rightarrow p_{2} \rightarrow \cdots \rightarrow p_{d-1} \rightarrow p_{d}=l
$$

along $g$ that leads to any point $l$ in $\mathcal{N}$, where the $p_{i}$ are in $\mathcal{N}$ and $p_{i-1} \neq p_{i}$. The path satisfies $a_{p_{i-1} p_{i}}>0$ for $i=1, \ldots, d$. It is then easy to construct a path along $g^{(n)}$ from any point $\left(k_{1}, \ldots, k_{n}\right) \in \mathcal{N}^{n}$ to any other point $\left(l_{1}, \ldots, l_{n}\right) \in \mathcal{N}^{n}$. We start by constructing a path from $\left(k_{1}, \ldots, k_{n}\right)$ to $\left(l_{1}, k_{2}, \ldots, k_{n}\right)$ using the irreducibility of $C_{A}$. Let

$$
k_{1}=p_{0} \rightarrow p_{1} \rightarrow p_{2} \rightarrow \cdots \rightarrow p_{d-1} \rightarrow p_{d}=l_{1}
$$

be a path along $g$ leading from $k_{1}$ to $l_{1}$ in $\mathcal{N}$. Then $a_{p_{i-1} p_{i}}>0$ for $i=1, \ldots, d$. From (3.1) we then see that

$$
a_{\left(p_{i-1}, k_{2}, \ldots, k_{n}\right),\left(p_{i}, k_{2}, \ldots, k_{n}\right)}^{(n)}=a_{p_{i-1} p_{i}}>0, \quad i=1, \ldots, d .
$$


Hence, the path

$$
\begin{aligned}
\left(k_{1}, \ldots, k_{n}\right)= & \left(p_{0}, k_{2}, \ldots, k_{n}\right) \rightarrow\left(p_{1}, k_{2}, \ldots, k_{n}\right) \rightarrow \cdots \\
& \rightarrow\left(p_{d-1}, k_{2}, \ldots, k_{n}\right) \rightarrow\left(p_{d}, k_{2}, \ldots, k_{n}\right) \\
= & \left(l_{1}, k_{2}, \ldots, k_{n}\right)
\end{aligned}
$$

links $\left(k_{1}, \ldots, k_{n}\right)$ to $\left(l_{1}, k_{2}, \ldots, k_{n}\right)$ along $g^{(n)}$.

We build similar paths from $\left(l_{1}, k_{2}, \ldots, k_{n}\right)$ to $\left(l_{1}, l_{2}, k_{3}, \ldots, k_{n}\right)$, etc., and finally from $\left(l_{1}, \ldots, l_{n-1}, k_{n}\right)$ to $\left(l_{1}, \ldots, l_{n}\right)$. The path from $\left(k_{1}, \ldots, k_{n}\right)$ to $\left(l_{1}, \ldots, l_{n}\right)$ along $g^{(n)}$ is the concatenation of all these paths.

Thus, $C_{A^{(n)}}$ is irreducible and $A^{(n)}$ satisfies condition (C2). This completes the proof.

The following theorem gives an expression for the $m^{n}$ when $A$ satisfies condition (C1) (which, as was remarked before, is equivalent to $A$ satisfying condition (C2)). The general case will be obtained in the next section, based on further analysis of the matrix $A$.

Theorem 3.1. Suppose that A satisfies condition $(C 1)$. Then $\left(m^{n}\right)_{n \in \mathbb{N}}$ is determined recursively by the relation

$$
m^{n}=\left(A^{(n)} \otimes I_{S}-I_{\mathcal{N}^{n}} \otimes Q^{*}\right)^{-1} \Lambda^{(n)} m^{n-1},
$$

with $m^{0}=(1, \ldots, 1)^{\top}$.

Proof. Note that, for all $u=\left(u_{1}, \ldots, u_{N}\right)^{\top} \in \mathbb{R}^{N}$ and $j \in S$, (2.3) reads

$$
\sum_{p \in \mathcal{N}}\left(\sum_{k \in \mathcal{N}} a_{k p} u_{k}\right) \partial_{p} \phi_{j}(u)=\left(\sum_{i \in \mathcal{N}} u_{i} \lambda^{i}(j)\right) \phi_{j}(u)+\sum_{k \in S} q_{j k}^{*} \phi_{k}(u) .
$$

Differentiating (3.5) with respect to the $l_{i}$ th variable, $i=1, \ldots, n$, and evaluating the expression at $u=(0, \ldots, 0)^{\top}$, yields

$$
\begin{aligned}
\sum_{p \in \mathcal{N}} \sum_{i=1}^{n} a_{l_{i}} \partial_{l_{1}} \cdots \partial_{l_{i-1}} \partial_{p} \partial_{l_{i+1}} \cdots \partial_{l_{n}} \phi_{j}(0)= & \sum_{i=1}^{n} \lambda^{l_{i}}(j) \partial_{l_{1}} \cdots \partial_{l_{i-1}} \partial_{l_{i+1}} \cdots \partial_{l_{n}} \phi_{j}(0) \\
& +\sum_{k \in S} q_{j k}^{*} \partial_{l_{1}} \cdots \partial_{l_{n}} \phi_{k}(0) .
\end{aligned}
$$

Since $\phi_{j}(u)=\mathrm{E}\left(\exp \left(\sum_{k=1}^{N} u_{k} W_{k}\right) \mid X^{*}(0)=j\right)$, we have

$$
\begin{aligned}
\partial_{l_{1}} \cdots \partial_{l_{i-1}} \partial_{p} \partial_{l_{i+1}} \cdots \partial_{l_{n}} \phi_{j}(0) & =\mathrm{E}\left(W_{l_{1}} \cdots W_{l_{i-1}} W_{p} W_{l_{i+1}} \cdots W_{l_{n}} \mid X^{*}(0)=j\right) \\
& =m_{j}^{n}\left(l_{1}, \ldots, l_{i-1}, p, l_{i+1}, \ldots, l_{n}\right) .
\end{aligned}
$$

In the same way,

$$
\begin{aligned}
\partial_{l_{1}} \cdots \partial_{l_{i-1}} \partial_{l_{i+1}} \cdots \partial_{l_{n}} \phi_{j}(0) & =m_{j}^{n-1}\left(l_{1}, \ldots, l_{i-1}, l_{i+1}, \ldots, l_{n}\right), \\
\partial_{l_{1}} \cdots \partial_{l_{n}} \phi_{k}(0) & =m_{k}^{n}\left(l_{1}, \ldots, l_{n}\right) .
\end{aligned}
$$

Thus, we have

$$
\begin{aligned}
\sum_{p \in \mathcal{N}} \sum_{i=1}^{n} a_{l_{i}} m_{j}^{n}\left(l_{1}, \ldots, l_{i-1}, p, l_{i+1}, \ldots, l_{n}\right)= & \sum_{i=1}^{n} \lambda^{l_{i}}(j) m_{j}^{n-1}\left(l_{1}, \ldots, l_{i-1}, l_{i+1}, \ldots, l_{n}\right) \\
& +\sum_{k \in S} q_{j k}^{*} m_{k}^{n}\left(l_{1}, \ldots, l_{n}\right) .
\end{aligned}
$$


This can be written in matrix form in the following way:

$$
\left(A^{(n)} \otimes I_{S}\right) m^{n}=\Lambda^{(n)} m^{n-1}+\left(I_{\mathcal{N}^{n}} \otimes Q^{*}\right) m^{n} .
$$

In order to obtain the final result, (3.4), it remains to show that the $\left(N^{n} \times K\right) \times\left(N^{n} \times K\right)$ matrix $M^{(n)}:=A^{(n)} \otimes I_{S}-I_{\mathcal{N}^{n}} \otimes Q^{*}$ is invertible.

From Lemma 3.2, $A^{(n)}$ satisfies condition (C2). This clearly implies that $A^{(n)} \otimes I_{S}$ satisfies condition (C1). Let $A^{(n)} \otimes I_{S}=D_{A^{(n)} \otimes I_{S}}-C_{A^{(n)} \otimes I_{S}}$ be the decomposition of $A^{(n)} \otimes I_{S}$. The matrix $I_{\mathcal{N}^{n}} \otimes Q^{*}$ has negative diagonal elements and nonnegative off-diagonal elements. We let $-\left(I_{\mathcal{N}^{n}} \otimes Q^{*}\right)=D_{I_{\mathcal{N}^{n}} \otimes Q^{*}}-C_{I_{\mathcal{N}^{n}} \otimes Q^{*}}$ be the decomposition of $-\left(I_{\mathcal{N}^{n}} \otimes Q^{*}\right)$.

Since $Q^{*}$ is the generator of a continuous-time Markov chain, we can easily check that the sum of the elements in any row of $I_{\mathcal{N}^{n}} \otimes Q^{*}$ is equal to 0 . Thus, since the sum of the elements in any row of $A^{(n)} \otimes I_{S}$ is nonnegative and the sum of the elements in one row of $A^{(n)} \otimes I_{S}$ is positive, $M^{(n)}$ has the same property.

The matrix $M^{(n)}$ has the decomposition $M^{(n)}=D_{M^{(n)}}-C_{M^{(n)}}$, with

$$
D_{M^{(n)}}=D_{A^{(n)} \otimes I_{S}}+D_{I_{\mathcal{N}^{n}} \otimes Q^{*}}, \quad C_{M^{(n)}}=C_{A^{(n)} \otimes I_{S}}+C_{I_{\mathcal{N}} n \otimes Q^{*}} .
$$

Let us now show that

$$
C_{M^{(n)}}:=\left(c_{\left(l_{1}, \ldots, l_{n}, j\right),\left(l_{1}^{\prime}, \ldots, l_{n}^{\prime}, j^{\prime}\right)}^{(n)}\right)_{\left(\left(l_{1}, \ldots, l_{n}, j\right),\left(l_{1}^{\prime}, \ldots, l_{n}^{\prime}, j^{\prime}\right)\right) \in\left(\mathcal{N}^{n} \times S\right)^{2}},
$$

where

$$
c_{\left(l_{1}, \ldots, l_{n}, j\right),\left(l_{1}^{\prime}, \ldots, l_{n}^{\prime}, j^{\prime}\right)}^{(n)}= \begin{cases}a_{\left(l_{1}, \ldots, l_{n}\right),\left(l_{1}^{\prime}, \ldots, l_{n}^{\prime}\right)}^{(n)} & \text { if } j=j^{\prime}, \\ q_{j j^{\prime}}^{*} & \text { if }\left(l_{1}, \ldots, l_{n}\right)=\left(l_{1}^{\prime}, \ldots, l_{n}^{\prime}\right) \text { and } j \neq j^{\prime}, \\ 0 & \text { otherwise, }\end{cases}
$$

is irreducible. Let us pick two distinct elements, $\left(r_{1}, \ldots, r_{n}, k\right)$ and $\left(s_{1}, \ldots, s_{n}, k^{\prime}\right)$, in $\mathcal{N}^{n} \times$ $S$ and prove that we can construct a path from $\left(r_{1}, \ldots, r_{n}, k\right)$ to $\left(s_{1}, \ldots, s_{n}, k^{\prime}\right)$ along the graph, $g^{M^{(n)}}$, corresponding to $C_{M^{(n)}}$. We begin by constructing a path from $\left(r_{1}, \ldots, r_{n}, k\right)$ to $\left(s_{1}, \ldots, s_{n}, k\right)$ of the form

$$
\begin{aligned}
\left(r_{1}, \ldots, r_{n}, k\right) & \rightarrow\left(p_{1}^{1}, \ldots, p_{n}^{1}, k\right) \rightarrow\left(p_{1}^{2}, \ldots, p_{n}^{2}, k\right) \rightarrow \cdots \\
& \rightarrow\left(p_{1}^{d-1}, \ldots, p_{n}^{d-1}, k\right) \rightarrow\left(s_{1}, \ldots, s_{n}, k\right)
\end{aligned}
$$

This is possible because

$$
c_{\left(l_{1}, \ldots, l_{n}, k\right),\left(l_{1}^{\prime}, \ldots, l_{n}^{\prime}, k\right)}^{(n)}=a_{\left(l_{1}, \ldots, l_{n}\right),\left(l_{1}^{\prime}, \ldots, l_{n}^{\prime}\right)}^{(n)}
$$

in (3.7) and because $A^{(n)}$ is irreducible. If $k=k^{\prime}$ then we construct the path from $\left(r_{1}, \ldots, r_{n}, k\right)$ to $\left(s_{1}, \ldots, s_{n}, k^{\prime}\right)$; otherwise, we finish by constructing a path from $\left(s_{1}, \ldots\right.$, $\left.s_{n}, k\right)$ to $\left(s_{1}, \ldots, s_{n}, k^{\prime}\right)$ of the form

$$
\begin{aligned}
\left(s_{1}, \ldots, s_{n}, k\right) & \rightarrow\left(s_{1}, \ldots, s_{n}, p_{1}\right) \rightarrow\left(s_{1}, \ldots, s_{n}, p_{2}\right) \rightarrow \cdots \\
& \rightarrow\left(s_{1}, \ldots, s_{n}, p_{h-1}\right) \rightarrow\left(s_{1}, \ldots, s_{n}, k^{\prime}\right) .
\end{aligned}
$$

This is possible because

$$
c_{\left(l_{1}, \ldots, l_{n}, j\right),\left(l_{1}, \ldots, l_{n}, j^{\prime}\right)}^{(n)}=q_{j j^{\prime}}^{*}
$$

in (3.7) and because $Q^{*}$ is irreducible. Thus, $C_{M^{(n)}}$ is irreducible.

The matrix $M^{(n)}$ then satisfies condition (C2). This implies that $M^{(n)}$ is invertible. We then easily recover (3.4) from (3.6). This completes the proof. 


\section{2. $A$ is a general matrix}

Theorem 3.1 deals with the case in which the sum of entries of a row of $A$ is nonnegative. We remove this strong assumption in this section. We thus again suppose that $A=\left(I-P^{\top}\right) D_{\mu}$, where $P$ is just an irreducible routeing matrix. There is then no reason why $A$ should satisfy condition $(\mathrm{C} 1)$ or condition (C2). However, we will find all of the moments of $W$ using results of Section 3.1.

Lemma 3.3. There exists a diagonal matrix, $H=\operatorname{diag}\left(v_{1}, \ldots, v_{N}\right)$ with $v_{i}>0$ for all $i=$ $1, \ldots, N$, such that $\hat{A}:=H^{-1} A H$ satisfies condition $(C 2)$.

The key thing to prove in this lemma is that $\hat{A}$ satisfies condition (C1). The fact that $\hat{A}$ satisfies condition (C2) is deduced from the fact that $P$ is irreducible.

Proof of Lemma 3.3. Let us remember that $A=\left(I-P^{\top}\right) D_{\mu}$. As was assumed in Section 1 , $P$ is a substochastic matrix, i.e. the sum of the entries in each row of $P$ is less than or equal to 1 and the sum of the entries in one row of $P$ is less than 1 . It is thus easy to verify that the sum of the entries in each row of $A^{\top}=D_{\mu}(I-P)$ is nonnegative and the sum of the entries in one row of $A^{\top}$ is positive.

Let $J$ be the diagonal matrix defined from $A^{\top}$ in the following way: an entry on the diagonal of $J$ is equal to the sum of the entries in the corresponding row of $A^{\top}$. Let $L$ be the difference between $J$ and $A^{\top}$, i.e.

$$
A^{\top}=J-L
$$

Since $P$ is substochastic, $J$ is a diagonal matrix with nonnegative coefficients and at least one positive coefficient, and $L$ is a matrix with nonpositive diagonal elements and nonnegative offdiagonal elements, with the sum of the coefficients in each row being equal to 0 . Furthermore, $L$ may be seen as the generator matrix of a continuous-time Markov chain, say $\{Y(t), t \in \mathbb{R}\}$. Moreover, $L$ is irreducible because $P$ is irreducible. Thus, $\{Y(t), t \in \mathbb{R}\}$ is ergodic and converges in distribution to some probability vector $v=\left(v_{1}, \ldots, v_{N}\right)$ with $v_{i}>0$.

Let $L^{*}$ be the generator matrix of the reversed chain, $\left\{Y^{*}(t), t \in \mathbb{R}\right\}$, of $\{Y(t), t \in \mathbb{R}\}$ (defined by $\left.Y^{*}(t):=Y\left((-t)^{-}\right)\right)$. Then $L^{*}$ satisfies $L^{*}=H^{-1} L^{\top} H$, with $H=\operatorname{diag}\left(v_{1}, \ldots, v_{N}\right)$.

Since $J, H$, and $H^{-1}$ are diagonal matrices, we have $H^{-1} J H=H^{-1} H J=J$, whence

$$
A=J-L^{\top}=J-H L^{*} H^{-1}=H\left(J-L^{*}\right) H^{-1} .
$$

Now, $L^{*}$ is, in particular, a generator matrix, and, thus, the sum of the entries in each of its rows is equal to 0 . Hence, $\hat{A}:=J-L^{*}=H^{-1} A H$ has the property that the sum of the entries in each of its rows is nonnegative and the sum of the entries in one of its rows is positive. Moreover, $\hat{A}$ is irreducible because $L^{*}$ is irreducible. Hence, $\hat{A}$ satisfies condition (C2).

Let us define the function $\bar{\lambda}:\{1, \ldots, K\} \rightarrow(0, \infty)^{N}$ by

$$
\bar{\lambda}(i)=\left(\bar{\lambda}^{1}(i), \ldots, \bar{\lambda}^{N}(i)\right)^{\top}=H^{-1} \lambda(i) .
$$

For all $n \in \mathbb{N}$, and similarly to $\Lambda^{(n)}$, let $\bar{\Lambda}^{(n)}$ be the $\left(N^{n} \times K\right) \times\left(N^{n-1} \times K\right)$ matrix defined blockwise by

$$
\bar{\Lambda}^{(n)}=\left(\bar{b}_{\left(l_{1}, \ldots, l_{n}\right),\left(k_{1}, \ldots, k_{n-1}\right)}^{(n)}\right)_{\left(\left(l_{1}, \ldots, l_{n}\right),\left(k_{1}, \ldots, k_{n-1}\right)\right) \in \mathcal{N}^{n} \times \mathcal{N}^{n-1}},
$$


where each $\bar{b}_{\left(l_{1}, \ldots, l_{n}\right),\left(k_{1}, \ldots, k_{n-1}\right)}^{(n)}$ is a $K \times K$ matrix defined, similarly to $b^{(n)}$, by

$$
\bar{b}_{\left(l_{1}, \ldots, l_{n}\right),\left(k_{1}, \ldots, k_{n-1}\right)}^{(n)}:= \begin{cases}\operatorname{diag}\left(\bar{\lambda}^{k_{i}}(1), \ldots, \bar{\lambda}^{k_{i}}(K)\right) & \text { if } l_{p}=k_{p} \text { for } p=1, \ldots, i-1 \\ & \text { and } l_{p+1}=k_{p} \text { for } p=i, \ldots, n-1, \\ 0 & \text { otherwise. }\end{cases}
$$

The following lemma is proved in the same way as Lemma 3.2, using Lemma 3.3.

Lemma 3.4. The matrix $\hat{A}^{(n)}$, defined in terms of $\hat{A}$ as $A^{(n)}$ is in terms of $A$ (see (3.1)), satisfies condition (C2).

Lemma 3.3 yields the following theorem, which is the central result of this paper.

Theorem 3.2. For all $n \in \mathbb{N}$ and $\left(l_{1}, \ldots, l_{n}\right) \in \mathcal{N}^{n}$, we have

$$
m^{n}\left(l_{1}, \ldots, l_{n}\right)=\frac{1}{\prod_{i=1}^{n} v_{l_{i}}} \hat{m}^{n}\left(l_{1}, \ldots, l_{n}\right)
$$

where the $\hat{m}^{n}$ are defined by the recursion

$$
\hat{m}^{n}=\left(\hat{A}^{(n)} \otimes I_{S}-I_{\mathcal{N}^{n}} \otimes Q^{*}\right)^{-1} \bar{\Lambda}^{(n)} \hat{m}^{n-1},
$$

with $\hat{m}^{0}=(1, \ldots, 1)^{\top}$.

Proof. From (1.2) we have

$$
\begin{aligned}
W & =\int_{0}^{\infty} \exp (-A s) \lambda\left(X^{*}(s)\right) \mathrm{d} s \\
& =\int_{0}^{\infty} \exp \left(-H \hat{A} H^{-1} s\right) \lambda\left(X^{*}(s)\right) \mathrm{d} s \\
& =\int_{0}^{\infty} H \exp (-\hat{A} s) H^{-1} \lambda\left(X^{*}(s)\right) \mathrm{d} s \\
& =H \hat{W},
\end{aligned}
$$

where

$$
\hat{W}=\int_{0}^{\infty} \exp (-\hat{A} s) H^{-1} \lambda\left(X^{*}(s)\right) \mathrm{d} s=\int_{0}^{\infty} \exp (-\hat{A} s) \bar{\lambda}\left(X^{*}(s)\right) \mathrm{d} s .
$$

From Lemma 3.4, $\hat{A}$ satisfies the assumption of Lemma 3.1. Let

$$
\hat{m}^{n}\left(l_{1}, \ldots, l_{n}\right):=\left(\hat{m}_{1}^{n}\left(l_{1}, \ldots, l_{n}\right), \ldots, \hat{m}_{K}^{n}\left(l_{1}, \ldots, l_{n}\right)\right)^{\top}
$$

where $\hat{m}_{i}^{n}\left(l_{1}, \ldots, l_{n}\right):=\mathrm{E}\left(\hat{W}_{l_{1}} \ldots \hat{W}_{l_{n}} \mid X^{*}(0)=i\right)$. From Theorem 3.1 , the $\hat{m}^{n}$ satisfy

$$
\hat{m}^{n}=\left(\hat{A}^{(n)} \otimes I_{S}-I_{\mathcal{N}^{n}} \otimes Q^{*}\right)^{-1} \bar{\Lambda}^{(n)} \hat{m}^{n-1},
$$

with $\hat{m}^{0}=(1, \ldots, 1)^{\top}$. Since

$$
m^{n}\left(l_{1}, \ldots, l_{n}\right)=\frac{\hat{m}^{n}\left(l_{1}, \ldots, l_{n}\right)}{\prod_{i=1}^{n} v_{l_{i}}},
$$

the proof is complete. 
To finish, we give the expression of the Laplace transform of $W$.

Proposition 3.1. For all $u=\left(u_{1}, \ldots, u_{N}\right) \in \mathbb{R}^{N}$, we have

$$
\phi(u)=\sum_{n=0}^{\infty} \sum_{\left(l_{1}, \ldots, l_{n}\right) \in \mathcal{N}^{n}} \frac{1}{l_{1} ! \cdots l_{n} !} \pi m^{n}\left(l_{1}, \ldots, l_{n}\right) u_{l_{1}} \cdots u_{l_{n}} .
$$

Proof. The proof follows simply from the fact that

$$
\exp \left(\sum_{i=1}^{N} u_{i} W_{i}\right)=\sum_{n=0}^{\infty} \sum_{\left(l_{1}, \ldots, l_{n}\right) \in \mathcal{N}^{n}} \frac{1}{l_{1} ! \cdots l_{n} !} W_{l_{1}} \cdots W_{l_{n}} u_{l_{1}} \cdots u_{l_{n}}
$$

and taking expectations in this equality.

\section{References}

[1] Asmussen, S. And Kella, O. (1996). Rate modulation in dams and ruin problem. J. Appl. Prob. 33, 523-535.

[2] Chen, H. And YaO, D. D. (2001). Fundamentals of Queueing Networks. Springer, New York.

[3] Graham, A. (1981). Kronecker Product and Matrix Calculus: with Applications. Halsted Press, New York.

[4] Kaspi, H. And Kella, O. (1996). Stability of feed-forward fluid networks with Lévy input. J. Appl. Prob. 33, 513-522.

[5] Kella, O. and Stadje, W. (2002). Exact results for a fluid model with state-dependent flow rates. Prob. Eng. Inf. Sci. 16, 389-402.

[6] Kella, O. And Stadje, W. (2002). Markov-modulated linear fluid networks with Markov additive input. J. Appl. Prob. 39, 413-420.

[7] Kella, O. ANd Whitt, W. (1996). Stability and structural properties of stochastic fluid networks. J. Appl. Prob. 33, 1169-1180.

[8] Kella, O. and Whitt, W. (1999). Linear stochastic fluid networks. J. Appl. Prob. 36, 244-260.

[9] Neuts, M. F. (1981). Matrix-Geometric Solutions in Stochastic Models. An Algorithmic Approach. Johns Hopkins University Press, Baltimore, MD.

[10] Rabehasaina, L. ANd Sericola, B. (2004). A second-order Markov-modulated fluid queue with linear service rate. J. Appl. Prob. 41, 758-777.

[11] Rabehasaina, L. And Sericola, B. (2003). Transient analysis of a Markov modulated fluid queue with linear service rate. In Proc. 10th Conf. Anal. Stoch. Modelling Tech. Appl. (ASMTA'03, Nottingham, June 2003), ed. D. Al-Dabass, SCS European Publishing House, pp. 234-239. 